\title{
The Expression Changes and Clinical Significance of Serum miRNA-1 in Patients with Heart Failure
}

\author{
BIN. JIAN, ZHEN LI, TAO. ZHANG, LI GUO. TAN AND CAN CAN QIANG* \\ Department of Cardiology, Renmin Hospital, Hubei University of Medicine, Shiyan, Hubei-442000, PR China
}

\section{Jian et al.: Changes and Significance of Serum miRNA-1 in Heart Failure}

\begin{abstract}
To investigate the expression changes and clinical significance of micro ribonucleic acid-1 in patients with heart failure. Ninety-four heart failure patients admitted to our hospital from December 2018 to December 2019 were selected as the study group and another 94 healthy check-ups in the same period were selected as the control group. The differences of micro ribonucleic acid-1 expression in the two study groups and its relationship with clinical indicators were compared and analyzed. The results showed that the plasma micro ribonucleic acid-1 expression level in the study group was higher and lower than that in the control group and the monocyte micro ribonucleic acid-1 expression level in the study group was lower than that in the control group and the difference was statistically significant $(p<0.05)$. The plasma and monocyte micro ribonucleic acid-1 expression levels decreased significantly with the increase of heart function grade in heart failure and the difference was statistically significant $(p<0.05)$. There was no statistically significant difference in plasma micro ribonucleic acid-1 expression levels among patients with different etiologies of heart failure $(p>0.05)$. Micro ribonucleic acid-1 expression levels were positively correlated with brain natriuretic peptide and left ventricular end diastolic diameter and negatively correlated with left ventricular ejection fraction $(\mathbf{p}<0.05)$. Micro ribonucleic acid-1, left ventricular ejection fraction and left ventricular end diastolic diameter were independent risk factors for heart failure, with statistically significant differences $(\mathbf{p}<\mathbf{0 . 0 5})$. The expression level of micro ribonucleic acid-1 in heart failure patients is reduced and the expression is more obvious with the increase of the disease, which is expected to be used as a marker for the diagnosis and judgment of heart failure.
\end{abstract}

Key words: Heart failure, miRNA-1, clinical significance, diagnostic index

Heart Failure (HF) is a group of syndromes with clinical manifestations of congestion in pulmonary circulation and systemic circulation and insufficient blood perfusion in organs and tissues due to various cardiac structural or functional diseases that lead to ventricular filling and impaired ejection function, insufficient cardiac output that fails to cover the metabolic requirements of tissues ${ }^{[1]}$. It is a disease that seriously endangers body's health and is responsible for the vast majority of cardiovascular diseases ${ }^{[2]}$. Heart disease such as Myocardial infarction, hypertension, aortic stenosis, valve disease can cause the changes of hemodynamics and neuroendocrine and these changes tend to cause pathological changes of myocardial intracellular molecules or transfer pathways. Under the action of pressure load, the heart is often manifested as hypertrophy, myocardial remodeling and other changes. Cardiac remodeling is the basic mechanism of $\mathrm{HF}$ and the pathophysiological basis of HF progression ${ }^{[3]}$.
Natriuretic peptide is a traditional biological marker of HF, which plays a very important role in clinical application. It can be divided into atrial natriuretic peptide (ANP) and B, C, D type natriuretic peptide, among which B-type natriuretic peptide is also called brain natriuretic peptide (BNP). Current studies suggest that BNP can directly reflect the prognosis of HF patients ${ }^{[4,5]}$ and natriuretic peptide can be used as the "gold standard" for the diagnosis and prognosis of HF patients. But in clinical application because of various reasons, they still exist many defects, plasma BNP concentration is affected by many diseases like kidney failure, lung disease, primary aldosteronism, thyroid disease, obesity, etc. its concentration also differs in people with different ages or genders ${ }^{[6,7]}$. Most BNP in the blood is metabolized in the kidney; its renal clearance rate will decrease with the renal function declines, which will cause the BNP metabolism to be blocked in the body and the concentration increase. The 
high volume state caused by decreased renal function can also lead to the change of BNP concentration in the circulation. Therefore, a more specific and valuable biomarker is needed to monitor the occurrence and development of HF.

With the deep understanding of the pathogenesis of HF, micro ribonucleic acid (miRNA) has been found to play a crucial role in the pathophysiology of HF and has important value in the diagnosis, condition, treatment effect evaluation and prognosis of HF. miRNA is a type of endogenous non-coding ribonucleic acid (RNA) consisting of approximately 19-25 nucleotides. The expression of specific genes can be regulated in a sequence-specific manner; this gene participates in almost all biological processes such as cell growth, differentiation, apoptosis and metabolism ${ }^{[8]}$. Recently, it has been discovered that microRNA is stably present in peripheral blood and can be used as a biomarker for disease ${ }^{[9]}$. In recent years, a number of studies have shown that changes in the levels of various miRNAs can be detected in $\mathrm{HF}^{[6]}$. In this study, serum miRNA-1 level in patients with HF was studied to explore its relationship with cardiac function and HF prognosis.

\section{MATERIALS AND METHODS}

\section{Clinical data:}

94 patients with HF admitted to our hospital from December 2018 to December 2019 were selected as the study group. Inclusion criteria: Confirmed as HF according to the diagnostic criteria in the Guidelines for The Diagnosis and Treatment of HF formulated by the Cardiovascular Branch of the Chinese Medical Association in 2007; The New York Heart Association (NYHA) heart function classification is at II-IV; Doppler echocardiography showed that its left ventricular ejection fraction (LVEF) was less than $50 \%$; The course of the disease was more than $1 \mathrm{y}$ and the condition was stable after treatment; No antiplatelet, anti-thrombotic drugs were taken within 2 $\mathrm{w}$; There is no blood relationship between included subjects; Signed informed consent.
Exclusion criteria: Patients with multiple organ failure; Patients with hematological diseases; Patients with malignant tumors; Patients with diabetes, systemic acute and chronic infections; Patients with abnormal liver function; Patients with hemorrhagic diseases. In addition, 94 healthy subjects in the same period were selected as the control group. The study group was $46-78 \mathrm{y}$ old and the control group was $47-76 \mathrm{y}$ old. There was no statistically significant difference between the two groups of general information $(p>0.05)$ and they were comparable as shown in Table 1.

\section{Identification of RNA extraction quality:}

\section{Peripheral blood plasma samples:}

$5 \mathrm{ml}$ peripheral blood extracted from venous was centrifuged at $3000 \mathrm{r} / \mathrm{min}$ to separate the plasma. Then the cell fragments in the plasma were separated at $1200 \mathrm{r} / \mathrm{min}$ and the nucleic acids on the cell fragments in the plasma were further separated at $25000 \mathrm{r} / \mathrm{min}$. The centrifuged plasma was stored in a refrigerator at $-80^{\circ}$ waiting for extraction. The RNA was extracted with the miRNEASY kit produced by Sigma company according to the instructions and fluorescent quantitative RNA reverse transcription combined with cDNA polymerase chain amplification technology (RT-PCR) were used for SNORD44 primer amplification and its quality identification. The extracted RNA was stored in the refrigerator at $-80^{\circ}$ for testing. Peripheral blood mononuclear cells samples: peripheral venous blood was mixed with Phosphate-buffered saline (PBS) solution at 1:1, spread the mixture on the lymphocyte separation solution, centrifuge it at $1500 \mathrm{r} / \mathrm{min}$, the second liquid layer was collected and re-suspended with PBS after 4 liquid layers appeared in the centrifuge tube and centrifuged twice at $1000 \mathrm{r} / \mathrm{min}$ to collect cells for RNA extraction.

\section{Real-time fluorescence quantitative RT-PCR reaction:}

The reaction was repeated 3 times for each sample using a 96 well reaction plate and their mean value was taken. The all-in-Pneumatic NAQRT-PCR assay kit

TABLE 1: COMPARISON OF GENERAL DATA BETWEEN STUDY GROUP AND CONTROL GROUP

\begin{tabular}{lcccc}
\hline Category & Control group & Research group & $\mathrm{t}$ & $\mathrm{P}$ \\
\hline Age(year) & $63.35 \pm 10.46$ & $64.22 \pm 11.57$ & 1.132 & 0.936 \\
Gender (Male/female) & $55 / 39$ & $57 / 37$ & 1.072 & 0.954 \\
Course of Disease (year) & $3.45 \pm 1.27$ & $3.52 \pm 1.43$ & 1.255 & 0.913 \\
BMI $\left(\mathrm{Kg} / \mathrm{cm}^{2}\right)$ & $23.57 \pm 2.36$ & $23.71 \pm 2.53$ & 1.437 & 0.872 \\
Proportion of drinking & $43(45.75 \%)$ & $44(46.81 \%)$ & 0.921 & 0.9661 \\
Smoking rate & $37(39.36)$ & $35(37.23 \%)$ & 1.732 & 0.788 \\
\hline
\end{tabular}


produced by Shanghai Biyantian company was used to amplify the miRNA with a Roche LightCycler 480II amplification analyzer. The details were as follows: pre-denaturation at $95^{\circ}$ for $10 \mathrm{~min}$, denaturation at $95^{\circ}$ for $10 \mathrm{~s}$, annealing at $60^{\circ}$ for $20 \mathrm{~s}$, amplification at $10 \mathrm{~s}$ at $75^{\circ}$ for 45 times, amplification at $75^{\circ}$ for $5 \mathrm{~min}$. MiRNA expression levels were determined by using $2^{-\triangle \Delta C T}$ calculated by RNU6-2.

\section{Observation indicators:}

Comparative analysis of the expression levels of miRNA-1 in peripheral blood and monocytes between the two groups. Compare the expression levels of miRNA-1 in peripheral blood and mononuclear cells of patients with different cardiac function classification in the study group. Compare the expression levels of miRNA-1 in patients with HF caused by coronary heart disease, rheumatic heart disease and dilated cardiomyopathy. Correlation between miRNA-1 expression level, BNP and left ventricular end diastolic diameter (LVEDD) and LVEF.

\section{Statistical analysis:}

SPSS 20.0 statistical software was used for data analysis. Analysis of variance and $\mathrm{T}$ test were used for measurement data. The $\chi^{2}$ test was used for count data. $\mathrm{p}<0.05$ was considered statistically significant.

\section{RESULTS AND DISCUSSION}

The results showed that the expression level of miRNA-1 in plasma of the study group was lower than that of the control group and the expression level of miRNA-1 in mononuclear cells of the study group was lower than that of the control group, with statistically significant differences $(p<0.05)$ as shown in fig. 1 . With the increase of heart function classification in $\mathrm{HF}$, the expression levels of miRNA-1 in plasma and monocytes of the patients were significantly decreased and the difference was statistically significant $(p<0.05)$ as shown in fig. 2 . There was no statistically significant difference in the expression level of miRNA-1 in plasma among patients with HF caused by different etiologies $(p>0.05)$ as shown in fig. 3 . The results showed that the expression level of miRNA-1 was positively correlated with BNP and LVEDD and negatively correlated with LVEF $(\mathrm{p}<0.05)$.
The results showed that miRNA-1, LVEF and LVEDD were independent risk factors for HF, with statistically significant differences $(\mathrm{p}<0.05)$, as shown in Table 2 .

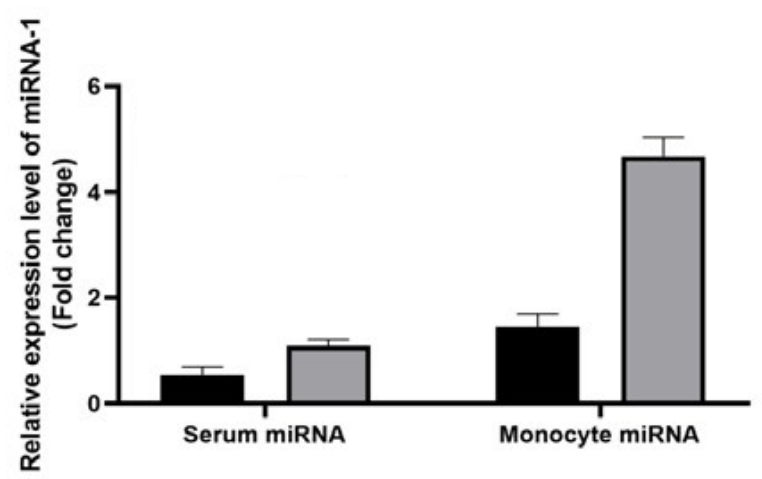

Fig. 1: Comparison of miRNA-1 expression levels in plasma and monocytes between the two groups ( $\square$ ): Experimental group ( $)$ : Control group

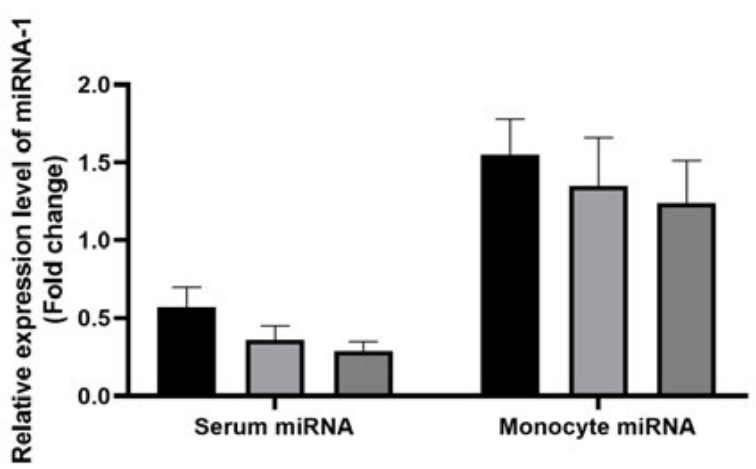

Fig. 2: Comparison of miRNA expression levels in peripheral blood and mononuclear cells of patients with different degrees

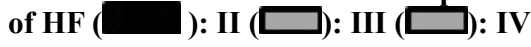

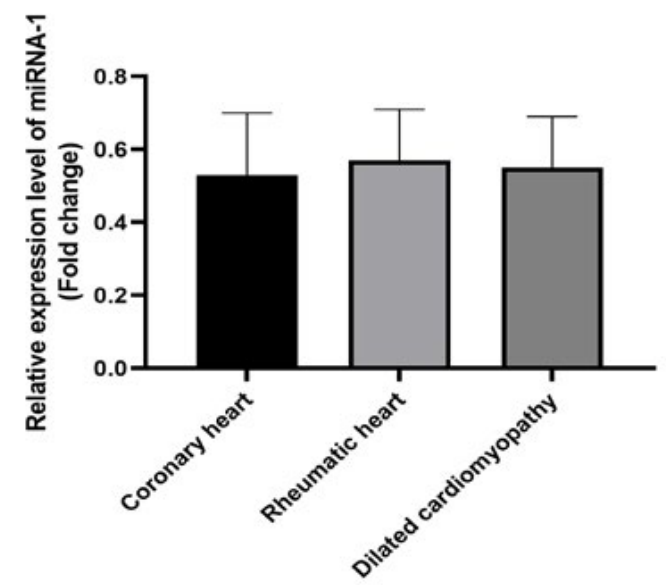

Fig. 3: Comparison of plasma miRNA expression levels in HF caused by different etiologies ( $\square$ ) Coronary heart ( $\square$ ) Rheumatic heart (־) Dilated cardiomyopathy

TABLE 2: RISK FACTOR ANALYSIS OF HF

\begin{tabular}{lcccccc}
\hline Variable & Partial regression coefficient & Standard error & Wald $\mathrm{X}^{2}$ value & $\mathrm{P}$ & OR value & $\mathbf{9 5 \% \mathrm { Cl }}$ \\
\hline miRNA-1 & 0.421 & 0.127 & 4.774 & 0.001 & 1.494 & $1.237 \sim 1.874$ \\
LVEDD & 0.386 & 0.133 & 3.953 & 0.006 & 1.378 & $1.201 \sim 1.943$ \\
LVEF & 0.374 & 0.158 & 4.114 & 0.002 & 1.417 & $1.156 \sim 1.793$ \\
\hline
\end{tabular}



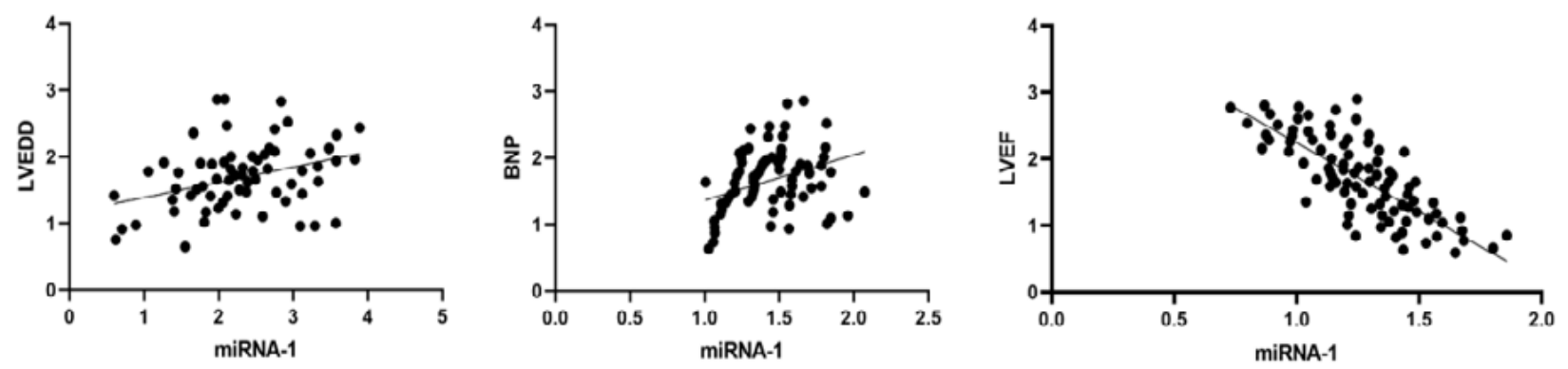

Fig. 4: The correlation between miRNA-1 expression level and BNP, left ventricular end diastolic diameter (LVEDD) and LVEF

With the aging of the population and the development of medical technology, the treatment level of myocardial infarction has increased and the prevalence of HF has been on the rise, which has become a serious threat to the health of middle-aged and elderly people. Patients with HF have ventricular filling and abnormal ejection functions, which are clinically manifested as fatigue, fluid retention and dyspnea ${ }^{[3,5]}$. HF can be divided into 4 stages, including pre-HF, pre-clinical HF, clinical $\mathrm{HF}$, refractory end-stage $\mathrm{HF}^{[10-12]}$. In clinical practice, plasma natriuretic peptide is mainly used as an auxiliary indicator for the diagnosis of HF, but the diagnostic effect is not ideal ${ }^{[13]}$.

MiRNA is a highly conserved non-coding small RNA with a long metabolic cycle and good stability, which is suitable for disease markers. MiRNAs can degrade mRNA of target genes or indirectly inhibit post-transcriptional translation of target genes by binding to specific paired sites, thus regulating gene expression levels. In addition, miRNAs can stimulate the expression of certain target genes ${ }^{[14,15]}$. Mi-RNAs have multiple biological functions. They are involved in a series of processes such as cell differentiation, proliferation and apoptosis and are closely related to hormone secretion, cardiovascular disease, diabetes and tumorigenesis ${ }^{[15-17]}$. Human miRNA-1 is associated with inflammation. In this study, the expression level of miRNA-1 in plasma and monocytes of HF patients in this research decreased compared with that in healthy people and the decrease is more obvious as the degree of HF worsens, suggesting that miRNA-1 may be released through damaged myocardial tissue and monocytes. The differential expression of miRNA-1 between patients with HF and healthy people makes it expected to be a marker for the antidiastole of HF.

In the process of clinical diagnosis and treatment, application of HF related biomarkers and is very vital for timely diagnosis. Zhang et al. ${ }^{[19]}$ showed that the overexpression of miRNA-1 was negatively correlated with LVEF in patients with myocardial infarction and speculated that miRNA-1 had a predictive value of the acute myocardial infarction occurrence in patients with HF. In clinical, the recommended markers for the evaluation of myocardial cell strain are mainly $\mathrm{BNP}^{[20]}$, which is mainly a polypeptide neurohormone secreted by ventricular myocytes under the stress state and transformed from the precursor of B-type natriuretic peptide $^{[21]}$. Myocardial ischemia and hypoxia can rapidly activate the natriuretic peptide system, increase the ventricular wall tension promote the secretion of BNP. BNP can bind to the natriuretic peptide receptor A to produce a series of effect like diuresis and sodium discharge increase, arterial vasodilation, inhibiting the growth of cardiovascular smooth muscle cells and inhibiting the ardiovascular remodeling. The level of BNP in plasma fluctuates with the disease state, which is obviously increased at the fact that the ventricular cavity is abnormally enlarged, the body volume load is increased or the excretion is reduced at the stage of renal failure. Therefore, BNP has a compensatory protection function for the body. But it is worth noting that some factors will affect the level of BNP, such as dysfunction in right ventricular and heart valve and pulmonary hypertension. At present, it is particularly important to explore new potential biomarkers for the diagnosis of HF, fully evaluation of the risk level of HF and to predict its prognosis.

In this study, plasma miRNA-1 expression level of HF patients was compared with BNP, LVEDD and LVEF, indicating that it was positively correlated with BNP and LVEDD and negatively correlated with LVEF, which was mutually corroborated with previous research. Combined with this study, it was further shown that plasma and monocyte miRNA-1 expression levels were low in HF patients, suggesting that changes in miRNA-1 level can reflect the degree of ventricular remodeling in HF patients and can be used as an important indicator to predict HF.

In summary, miRNA has the characteristics of high stability. The expression level of miRNA-1 shows 
a downward trend in patients with HF, which is significantly different from healthy people. The expression level of miRNA-1 is related to BNP, LVEDD and LVEF in patients with HF, suggesting it is a independent risk factors for HF, thus miRNA-1 is expected to be a marker for the diagnosis and evaluation of HF. However, due to high cost, multiple cycles and low concentration, miRNA detection is prone to large errors. Meanwhile, miRNA is easy to be degraded, which is tending to cause inconsistency between detection results and reality ${ }^{[20,21]}$. Besides, the role of miRNA in diseases is not fully understood and many problems still need to be further studied and resolved.

\section{Conflicts of interest}

The authors report no conflicts of interest.

\section{Acknowledgements}

This work was supported by Department of Cardiology, Renmin Hospital, Hubei University of Medicine, Shiyan ,Hubei 442000, PR China. BIN. JIAN and ZHEN. LI contributed equally to this work.

\section{REFERENCES}

1. Xiao N, Zhang J, Chen C, Wan Y, Wang N, Yang J. miR-129$5 \mathrm{p}$ improves cardiac function in rats with chronic heart failure through targeting HMGB1. Mamm Genome 2019;30(9):27688 .

2. Raso A, Dirkx E, Philippen LE, Fernandez-Celis A, De Majo F, Sampaio-Pinto V, et al. Therapeutic delivery of miR-148a suppresses ventricular dilation in heart failure. Mol Ther 2019;27(3):584-99.

3. He R, Ding C, Yin P, He L, Xu Q, Wu Z, et al. MiR-1a-3p mitigates isoproterenol-induced heart failure by enhancing the expression of mitochondrial ND1 and COX1. Exp Cell Res 2019;378(1):87-97.

4. Schulte C, Barwari T, Joshi A, Theofilatos K, Zampetaki A, Barallobre-Barreiro J, et al. Comparative analysis of circulating noncoding RNAs versus protein biomarkers in the detection of myocardial injury. Circ Res 2019;125(3):328-40.

5. Qiao L, Hu S, Liu S, Zhang $\mathrm{H}, \mathrm{Ma} \mathrm{H}$, Huang $\mathrm{K}$, et al. microRNA-21-5p dysregulation in exosomes derived from heart failure patients impairs regenerative potential. J Clin Invest 2019;129(6):2237-50.

6. Huang YM, Li WW, Wu J, Han M, Li BH. The diagnostic value of circulating microRNAs in heart failure. Exp Ther Med 2019;17(3):1985-2003.

7. Lin $X$, Zhang S, Huo Z. Serum circulating miR-150 is a predictor of post-acute myocardial infarction heart failure. Int Heart J 2019;60(2):280-6.

8. Li H, Fan J, Zhao Y, Zhang X, Dai B, Zhan J, et al. Nuclear miR-320 mediates diabetes-induced cardiac dysfunction by activating transcription of fatty acid metabolic genes to cause lipotoxicity in the heart. Circ Res 2019;125(12):1106-20.
9. Pinchi E, Frati P, Aromatario M, Cipolloni L, Fabbri M, La Russa R, et al. miR-1, miR-499 and miR-208 are sensitive markers to diagnose sudden death due to early acute myocardial infarction. J Cell Mol Med 2019;23(9):6005-16.

10. Wüst S, Dröse S, Heidler J, Wittig I, Klockner I, Franko A, et al. Metabolic maturation during muscle stem cell differentiation is achieved by miR-1/133a-mediated inhibition of the Dlk1Dio3 mega gene cluster. Cell Metabol 2018;27(5):1026-39.

11. Cho Y, Baldan A. Quest for new biomarkers in atherosclerosis. Mol Med 2013;110(4): 325-30.

12. Li Q, Song XW, Zou J, Wang GK, Kremneva E, Li XQ, et al. Attenuation of microRNA-1 derepresses the cytoskeleton regulatory protein twinfilin-1 to provoke cardiac hypertrophy. J Cell Sci 2010;123(14):2444-52.

13. Wang J, Huang W, Xu R, Nie Y, Cao X, Meng J, et al. MicroRNA-24 regulates cardiac fibrosis after myocardial infarction. J Cell Mol Med 2012;16(9):2150-60.

14. Castoldi G, Di Gioia CR, Bombardi C, Catalucci D, Corradi B, Gualazzi MG, et al. MiR-133a regulates collagen 1A1:potential role of miR-133a in myocardial fibrosis in angiotensin IIdependent hypertension. J Cell Physiol 2012;227(2):850-6.

15. Huang F, Li ML, Fang ZF, Hu XQ, Liu QM, Liu ZJ, et al. Overexpression of MicroRNA-1 improves the efficacy of mesenchymal stem cell transplantation after myocardial infarction. Cardiology 2013;125(1):18-30.

16. Duisters RF, Tijsen AJ, Schroen B, Leenders JJ, Lentink V, van der Made I, et al. miR-133 and miR-30 regulate connective tissue growth factor: implications for a role of microRNAs in myocardial matrix remodeling. Circ Res 2009;104(2):170-8.

17. Bonauer A, Carmona G, Iwasaki M, Mione M, Koyanagi $\mathrm{M}$, Fischer A, et al. MicroRNA-92a controls angiogenesis and functional recovery of ischemic tissues in mice. Science 2009;324(5935):1710-3.

18. Fasanaro P, Greco S, Lorenzi M, Pescatori M, Brioschi M, Kulshreshtha R, et al. An integrated approach for experimental target identification of hypoxia-induced miR-210. J Biol Chem 2009;284(50):35134-43.

19. Zhou J, Wang KC, Wu W, Subramaniam S, Shyy JY, Chiu JJ, et al. MicroRNA-21 targets peroxisome proliferators-activated receptor-alpha in an autoregulatory loop to modulate flowinduced endothelial inflammation. Proc Natl Acad Sci U S A 2011;108(25):10355-60.

20. Weber M, Baker MB, Moore JP, Searles CD. MiR-21 is induced in endothelial cells by shear stress and modulates apoptosis and e NOS activity. Biochem Biophys Res Commun 2010;393(4):643-8.

21. Yu XY, Song YH, Geng YJ, Lin QX, Shan ZX, Lin SG, et al. Glucose induces apoptosis of cardiomyocytes via microRNA-1 and IGF-1. Biochem Biophys Res Commun 2008;376(3):54852.

This is an open access article distributed under the terms of the Creative Commons Attribution-NonCommercial-ShareAlike 3.0 License, which allows others to remix, tweak, and build upon the work non-commercially, as long as the author is credited and the new creations are licensed under the identical terms

This article was originally published in a special issue, "Clinical Research in Pharmaceutical and Biomedical Sciences" Indian J Pharm Sci 2021:83(1)Spl issue1;111-115 